\title{
Communication \\ Estimation of the Abduction/Adduction Movement of the Metacarpophalangeal Joint of the Thumb
}

\author{
Néstor J. Jarque-Bou *(D), Margarita Vergara (1) and Joaquín L. Sancho-Bru $(\mathbb{C}$ \\ Department of Mechanical Engineering and Construction, Universitat Jaume I, E12071 Castellón, Spain; \\ vergara@uji.es (M.V.); sancho@uji.es (J.L.S.-B.) \\ * Correspondence: jarque@uji.es; Tel.: +34-(96)-4728125
}

Citation: Jarque-Bou, N.J.; Vergara, M.; Sancho-Bru, J.L. Estimation of the Abduction/Adduction Movement of the Metacarpophalangeal Joint of the Thumb. Appl. Sci. 2021, 11, 3158. https://doi.org/10.3390/app11073158

Academic Editor: Chi-Seung Lee

Received: 25 February 2021

Accepted: 26 March 2021

Published: 1 April 2021

Publisher's Note: MDPI stays neutral with regard to jurisdictional claims in published maps and institutional affiliations.

Copyright: (c) 2021 by the authors. Licensee MDPI, Basel, Switzerland. This article is an open access article distributed under the terms and conditions of the Creative Commons Attribution (CC BY) license (https:/ / creativecommons.org/licenses/by/ $4.0 /)$.

\begin{abstract}
Thumb opposition is essential for grasping, and involves the flexion and abduction of the carpometacarpal and metacarpophalangeal joints of the thumb. The high number of degrees of freedom of the thumb in a fairly small space makes the in vivo recording of its kinematics a challenging task. For this reason, along with the very limited independence of the abduction movement of the metacarpophalangeal joint, many devices do not implement sensors to measure such movement, which may lead to important implications in terms of the accuracy of thumb models. The aims of this work are to examine the correlation between thumb joints and to obtain an equation that allows thumb metacarpophalangeal abduction/adduction movement to be estimated from the other joint motions of the thumb, during the commonest grasps used during activities of daily living and in free movement. The correlation analysis shows that metacarpophalangeal abduction/adduction movement can be expressed mainly from carpometacarpal joint movements. The model thus obtained presents a low estimation error $\left(6.29^{\circ}\right)$, with no significant differences between grasps. The results could benefit most fields that do not typically include this joint movement, such as virtual reality, teleoperation, 3D modeling, prostheses, and exoskeletons.
\end{abstract}

Keywords: regression analysis; grasping; free movement; thumb kinematics; joint estimation

\section{Introduction}

The thumb is essential for grasping, as it leads the motion of the other digits [1]. The opposition of the thumb to the fingers is essential for grasping [2] and is part of most grasp positions needed to carry out activities of daily living (ADL) [3]. From the mechanical point of view, this complex movement is performed mainly at the thumb metacarpophalangeal (MCP) and carpometacarpal (CMC) joints and involves several motions: thumb CMC flexion and abduction, and thumb MCP flexion and abduction.

The thumb MCP joint is of the condylar type, whereas the thumb CMC joint is a saddle joint, both of them having two main degrees of freedom (DoF): flexion-extension and abduction/adduction, defined as rotations around two non-perpendicular and nonintersecting axes on each joint [4]. At the MCP joint, the flexion-extension axis is in the metacarpal passing under the epicondyles, and the abduction/adduction axis is in the proximal phalanx, passing between the sesamoids just proximal to its beak. At the CMC joint, the flexion/extension axis is in the trapezium and the abduction/adduction axis is in the metacarpal, their positions being constant throughout the range of motion of the joint, and not perpendicular to the bones [5]. As the axes are non-perpendicular and non-intersecting, it is difficult to assign classic planes to thumb movements and postures, leading to different ways of defining the thumb motion [6]. For this reason, some studies also consider pronation at the CMC joint, although the most widely accepted kinematic model is to use only five DoF: CMC flexion-extension and abduction/adduction, MCP flexion-extension and abduction/adduction, and interphalangeal (IP) flexion-extension.

Interestingly, all the muscles that are abductors or adductors of the MCP joint are also abductors or adductors of the CMC joint. This sophisticated biomechanical structure 
underlies a dimensionality reduction mechanism during motion control due to the existence of intra-thumb muscular synergies [7]. This actuation linkage, together with the tendinous configuration of the MCP joint, results in a very limited capacity for independent abduction of the MCP joint, which means that movements of the thumb MCP joint are closely linked to the movements of the thumb CMC joint [4,8]. In this regard, Li and Tang [8] analyzed the global coordination of the thumb during free movements (opposition and circumduction), which revealed coordination between the flexion and pronation at the CMC joint, and between the flexion of the CMC joint and the MCP joint. However, no previous work has studied the correlation between thumb joints during the common grasps used to perform ADL.

In addition, the high number of thumb DoF in a fairly small space makes it a challenging task to record in vivo joint rotations accurately. As a consequence, many devices used to measure the kinematics of the hand (such as instrumented gloves, e.g., Cyberglove) do not implement sensors to measure the MCP abduction movement [9], and many kinematic studies in the literature do not consider this movement, although in some cases they do use devices capable of measuring it $[10,11]$. This approximation may lead to important implications for the accuracy of biomechanical models [12,13].

This work has two aims: (1) to examine the correlation between thumb joints during grasps, and (2) to obtain the equation that allows the thumb MCP abduction/adduction movement to be estimated from the other joint motions of the thumb during the commonest grasps used during ADL.

\section{Materials and Methods}

All the experiments performed for this study were authorized by the Ethical Committee of the University Jaume I (Castellón, Spain), in June 2019 (Process No. 31/2019).

\subsection{Experiment A. Prediction Model}

\subsubsection{Experimental Procedure}

Seventeen healthy-handed subjects participated in the experiments: 10 women and 7 men (Mean \pm SD; age: $36.8 \pm 9.5$ years; hand length: $187.3 \pm 12.0 \mathrm{~mm}$; hand width: $78.4 \pm 5.7 \mathrm{~mm}$ ). The subjects were asked to grasp and transport diverse everyday objects (Figure 1) using the grasp types most commonly employed in ADL [14] (Table 1): Lumbrical grasp (Figure 1c), Oblique-palmar grasp (Figure 1d), Five-finger pinch grasp (Figure 1e), and Cylindrical grasp (Figure 1f). Each grasp type was tested with commonly manipulated objects with a range of shapes, sizes, textures, stiffnesses and weights (Table 1). Participants were asked to use different grasps and objects, so as to have 9-10 trials per combination (Table 1).

Subjects were seated by a table and, after familiarization with the objects, moved each object from point $B$ to point $C$ (Figure $1 b$ ), starting with the hand resting at point A (Figure 1a) and the object at point $B$. This process was repeated three times for each type of grasp and object. The order of grasps for each subject was randomized. Finally, the total numbers of postures recorded for each grasp were 162 (Lumbrical grasp), 108 (Oblique-palmar grasp), 210 (Five-finger grasp), and 180 (Cylindrical grasp). 


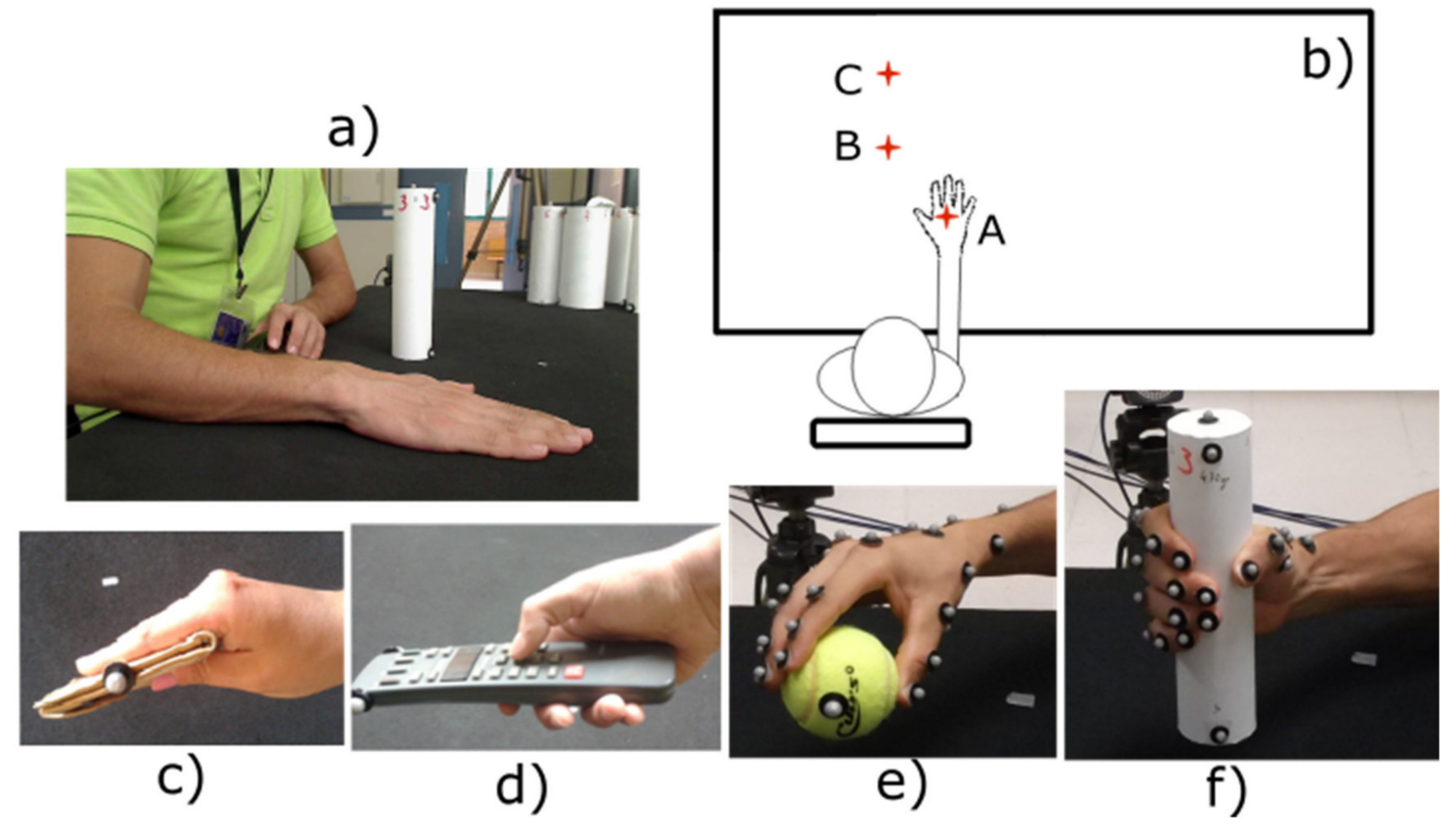

Figure 1. Top: (a) starting posture; (b) final and initial posture of the hand (A) on the table, and initial (B) and final (C) positions of the object. Bottom: Lumbrical grasp (c), Oblique-palmar grasp (d), Five-finger grasp (e), and Cylindrical grasp (f).

Table 1. Objects, properties (weight in grams (g) and size in millimeters ( $\mathrm{mm})$ ), subjects and types of grasps performed during the postures recorded in the experiment.

\begin{tabular}{|c|c|c|c|c|}
\hline Object & Weight (g) & Size (mm) & Subjects & Grasps \\
\hline Heavy/thin cylinder & 470 & $35 \varnothing$ & $\begin{array}{c}9 \\
10 \\
10 \\
9\end{array}$ & $\begin{array}{c}\text { Lumbrical } \\
\text { Cylindrical } \\
\text { Five-finger } \\
\text { Oblique-palmar }\end{array}$ \\
\hline Heavy/medium cylinder & 470 & $50 \varnothing$ & $\begin{array}{c}9 \\
10 \\
10\end{array}$ & $\begin{array}{l}\text { Lumbrical } \\
\text { Cylindrical } \\
\text { Five-finger }\end{array}$ \\
\hline Heavy/thick cylinder & 470 & $65 \varnothing$ & $\begin{array}{c}9 \\
10 \\
10\end{array}$ & $\begin{array}{l}\text { Lumbrical } \\
\text { Cylindrical } \\
\text { Five-finger }\end{array}$ \\
\hline Light/medium cylinder & 193 & $50 \varnothing$ & $\begin{array}{l}10 \\
10\end{array}$ & $\begin{array}{l}\text { Cylindrical } \\
\text { Five-finger }\end{array}$ \\
\hline Sphere & 251 & 76 & 9 & Lumbrical \\
\hline Wallet & 40.5 & 120 & 9 & Lumbrical \\
\hline Remote controller & 74 & $450 \times 180$ & 9 & Oblique-palmar \\
\hline Lighter & 100 & $510 \times 330$ & 9 & Oblique-palmar \\
\hline Spoon & 33 & $40 \times 230$ & 9 & Oblique-palmar \\
\hline Glass & 130 & $80 \varnothing \times 95$ & $\begin{array}{l}10 \\
10\end{array}$ & $\begin{array}{l}\text { Cylindrical } \\
\text { Five-finger }\end{array}$ \\
\hline Tennis ball & 100 & 70 & $\begin{array}{l}10 \\
10\end{array}$ & $\begin{array}{l}\text { Cylindrical } \\
\text { Five-finger }\end{array}$ \\
\hline Marker pen & 30 & $10 \times 145$ & 10 & Five-finger \\
\hline Book & 158 & $35 \times 105 \times 150$ & 9 & Lumbrical \\
\hline
\end{tabular}


Hand posture (23 joint angles) was recorded by tracking the location of 29 markers on the subjects' hands using a Vicon motion capture system [15]. For this study, only the kinematic data of the thumb joints were considered: flexion of the interphalangeal joint (IPF), flexion and abduction of the MCP joint (MCPF and MCPA, respectively) and flexion and abduction of the CMC joint (CMCF and CMCA, respectively). One neutral posture was used as a reference (for comparison of measures) that indicated zero for all the thumb angles: the thumb resting, with the fingers flexed and in a relaxed posture, on the lateral side of the middle finger (Figure 2). The grasping postures considered in the kinematic analyses carried out were those corresponding to the instant in which the object was at the highest position while being transported.

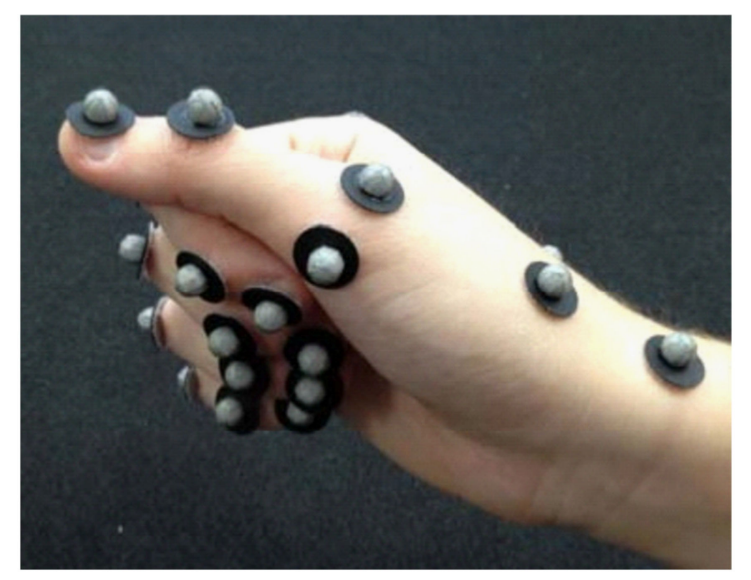

Figure 2. Reference posture for the thumb angles.

\subsubsection{Data Processing and Statistical Analysis}

Each observation (grasping posture) consisted of a row vector of 5 variables (the thumb joint angles). The thumb kinematics coordination was assessed by means of the data correlation matrix of all the thumb joint angles (matrix $5 \times 5$ ) for all grasps (considering all 660 postures recorded). The correlation coefficient was computed using Pearson's correlation. The joint angles that were most correlated with MCP abduction/adduction movement (referred to from now on as representative angles) were used as predictors of its movement in the next section.

It is proposed that the thumb MCP abduction/adduction angle for a given frame be expressed as a combination of the representative angles recorded for the same frame. In order to obtain the model that minimizes prediction error while employing the smallest number of variables, a sequential search method was applied [16]. In particular, a stepwise multiple regression analysis (MATLAB function stepwiselm) was used, including all the recorded postures (660). This function finds the best regression estimates based on the forward addition and backward elimination of variables in the equation, which is one of the most preferred methods [16]. As a criterion for adding or removing terms, a $p$-value for an F-test of the change in the sum of the squared error of less than 0.1 was selected [16]. The model for the regression selected was "interactions", which contains an intercept, linear terms of each predictor, and all the products of pairs of distinct predictors (without squared terms). As a result, the prediction model was presented, and then the estimation errors (Root mean square errors-RMSE) that derived from its application were studied. Finally, a one-way ANOVA was performed to check for significant differences in the errors for each grasp type.

\subsection{Experiment B. Evaluation of the Goodness of the Regression}

\subsubsection{Experimental Procedure}

The goodness of the regression was evaluated against two different kinematic datasets: (1) reach-to-grasp movements from 226 trials in experiment A: 9 subjects, reach-to-grasp 
movement of seven objects (book, cylinders, tennis ball and wallet, 3 repetitions of each), 26.499 frames altogether; and (2) grasping postures different from those used for the regression: 5 subjects, 7 cylinders of different sizes, 4 grasp types (cylindrical, five-finger, three-finger, two-finger grasp), 3 repetitions of each, with 525 postures altogether. In both cases, the thumb joint angles were recorded with the same methodology used in Experiment A, using the same protocol to obtain anatomical angles and the same filtering.

\subsubsection{Data Processing and Statistical Analysis}

By using the prediction model (namely, the regression model described in 2.1.2), thumb MCP abduction/adduction joint angles were estimated for each frame of the two sets from experiment B. Global root mean square errors (RMSEs) between estimated and recorded joint angles were obtained for both sets, calculated across subjects, frames, and movements/postures.

\section{Results}

\subsection{Experiment A. Prediction Model}

Figure 3 shows the statistics of the data recorded in Experiment A. All joint angles presented wide ranges across postures, covering their reported range of motion $[9,17]$. Note that $\mathrm{CMC}$ abduction/adduction, MCP flexion/extension and MCP abduction/adduction angles presented more negative than positive values, i.e., the postures recorded needed the thumb more abducted and the MCP extended.

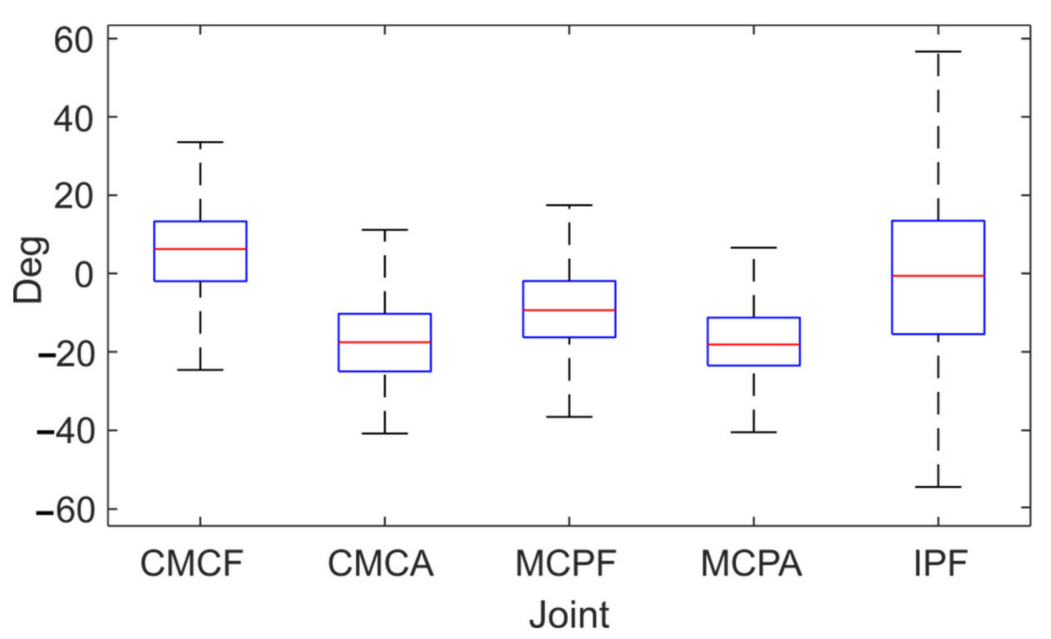

\begin{tabular}{c|cc} 
Joint & Range (deg) & $\mu(\mathrm{deg})$ \\
\hline CMCF & 75.45 & 5.24 \\
CMCA & 72.34 & -16.15 \\
MCPF & 64.64 & -9.22 \\
MCPA & 51.92 & -16.66 \\
IPF & 134.68 & -0.82
\end{tabular}

Figure 3. Left: Box plot with angles recorded across postures. The horizontal marks in the center of the boxes are the median; the edges of the boxes are the 25th and 75th percentile; whiskers extend to 1.5 times the interquartile range. Right: range (maximum minus minimum) and average values ( $\mu$ ) of each joint movement. Positive values represent flexion and adduction angles; negative values represent extension and abduction angles. CMC abduction/adduction (CMCA) and flexion/extension (CMCF); MCP abduction/adduction (MCPA) and flexion/extension (MCPF); and IP flexion/extension (IPF).

Figure 4 shows the correlations between the thumb joint angles for all the postures (660) performed in Experiment A. MCP abduction/adduction joint movement presented the highest correlation of values and was positively correlated with CMC abduction/adduction (0.68) and with MCP flexion/extension (0.27) joint movement. Conversely, MCP abduction/adduction joint movement was negatively correlated with CMC flexion/extension $(-0.30)$ joint movement. A negative correlation between IP and CMC joints was also present. The MCP flexion/extension appeared to be poorly coordinated with the rest of the thumb joints. 

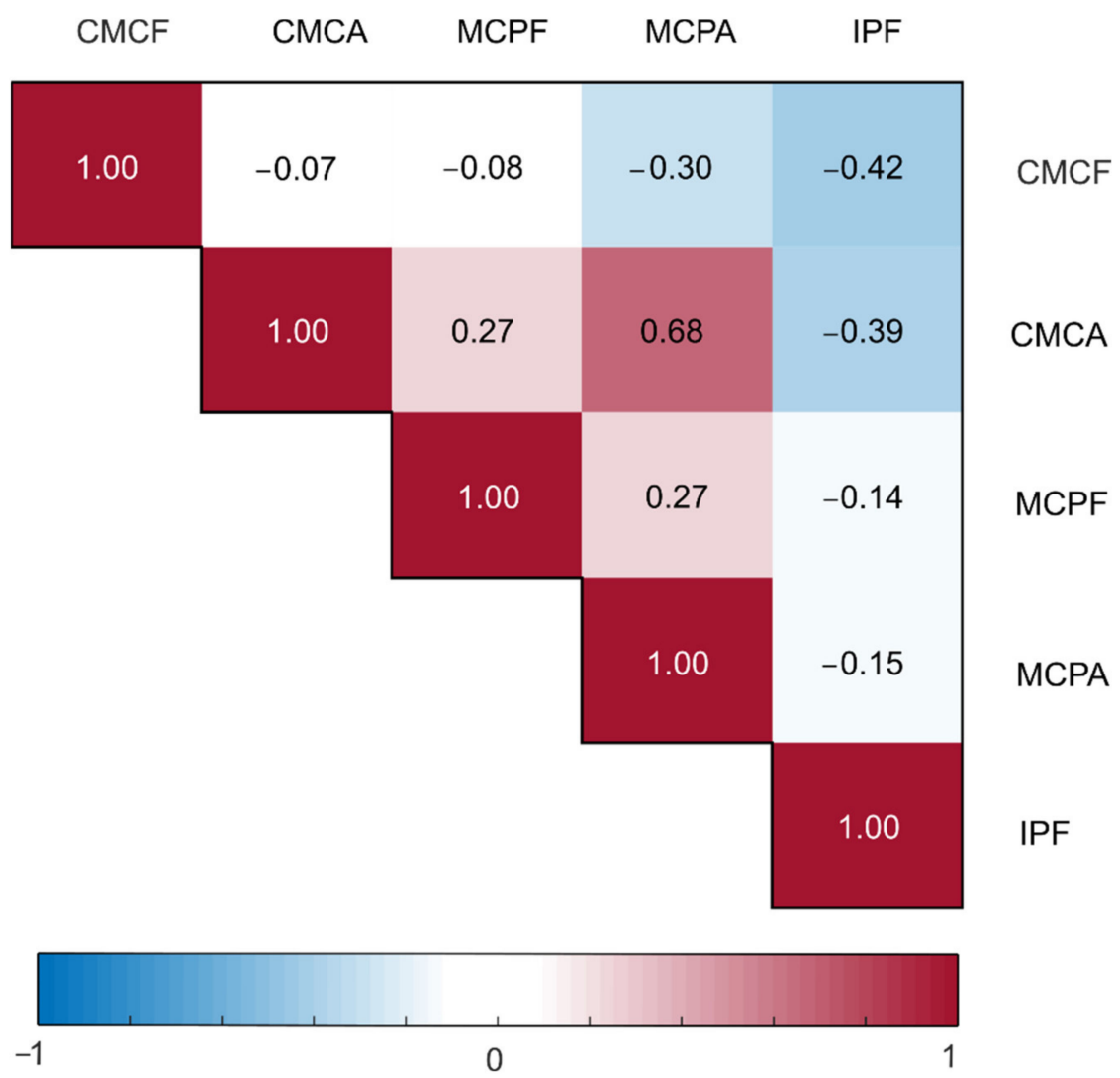

Figure 4. Correlation coefficients between thumb joints movements (CMC abduction/adduction (CMCA) and flexion/extension (CMCF), MCP abduction/adduction (MCPA) and flexion/extension (MCPF) and IP flexion/extension (IPF)) for all the postures performed.

CMC abduction/adduction is the only joint movement added to the regression model as an independent variable. Interaction between CMC flexion/extension and CMC abduction/adduction is also present in the model. Table 2 shows the coefficients for the prediction model to estimate the MCP abduction/adduction angles. $p$-value indicate that all the coefficients are significant $(p$-value $<0.05)$. Equation $(1)$ presents the regression equation obtained for the estimation of the MCP abduction/adduction joint movement (with joint angles in degrees).

$$
\mathrm{MCPA}_{\mathrm{est}_{\mathrm{i}}}=-5.825+0.575 \times \mathrm{CMCA}_{\mathrm{i}}+0.015 \times \mathrm{CMCF}_{\mathrm{i}} \times \mathrm{CMCA}_{\mathrm{i}}
$$

Table 2. Coefficients, standard error of the coefficients (SE), t-statistic for each coefficient (tStat) and p-value for the t-statistic of the hypothesis test whether the corresponding coefficient, obtained for MCP abduction/adduction estimation, is equal to zero or not ( $p$-value). CMCA: CMC abduction/adduction; CMCF: CMC flexion/extension.

\begin{tabular}{ccccc}
\hline & Coefficient & SE & tStat & $p$-Value \\
\hline Intercept & -5.825 & 0.428 & -13.622 & 0.000 \\
CMCA & 0.575 & 0.021 & 27.836 & 0.000 \\
CMCF $\times$ CMCA & 0.015 & 0.001 & 10.584 & 0.000 \\
\hline
\end{tabular}

Figure 5 shows the interaction effect between CMC flexion/extension and CMC abduction/adduction. In particular, it shows that the relationship between CMC flexion/extension and $\mathrm{MCP}$ abduction/adduction changes direction based on the $\mathrm{CMC}$ abduction/adduction values. The curves of the effect estimated with the other predictor fixed at extreme values are not parallel, thus corroborating interactions between the predictors: for 
high positive values of CMC abduction/adduction, there is a positive relationship between MCP abduction/adduction and CMC flexion/extension, while for high negative values the relationship is negative.

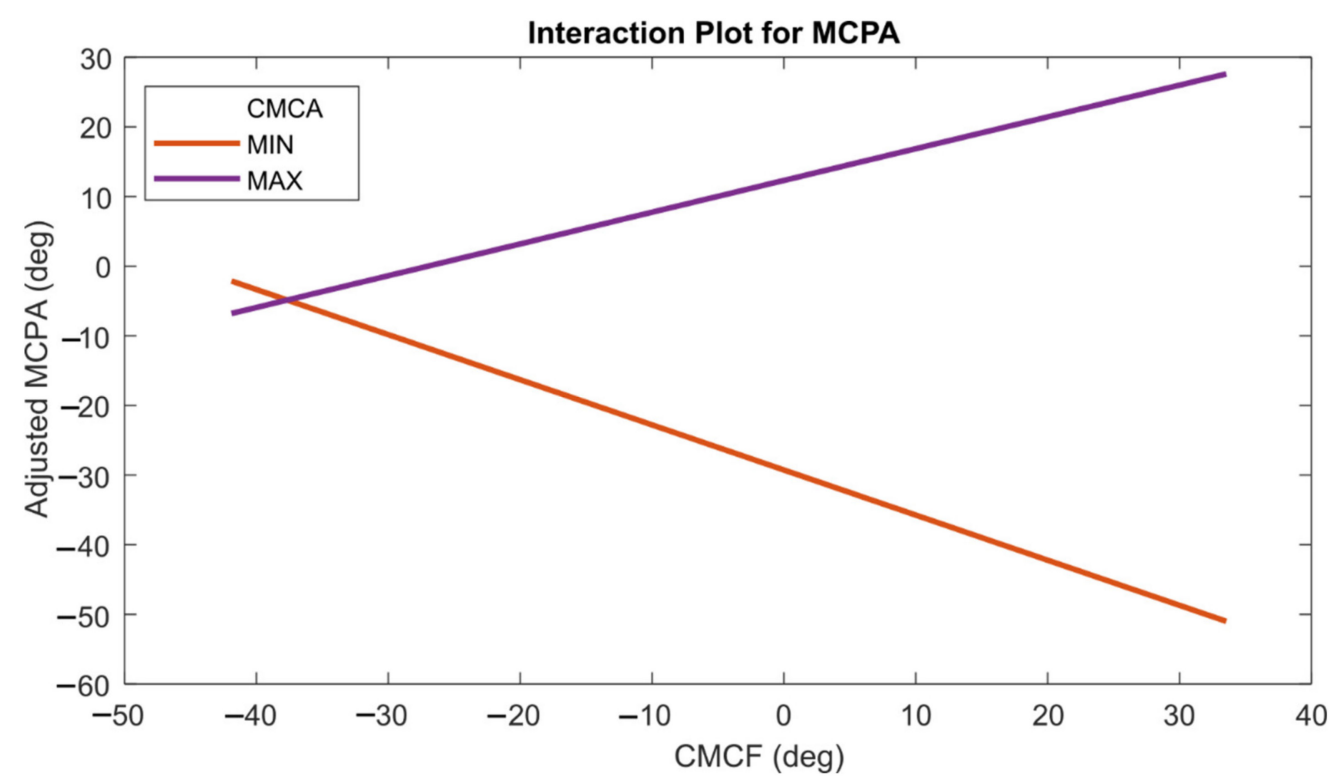

Figure 5. Interaction effect between CMC flexion/extension (CMCF) and CMC abduction/adduction (CMCA) for the estimation of MCP abduction/adduction (MCPA). The estimated effect with the other predictor fixed at extreme values (maximum (MAX; $\left.31.55^{\circ}\right)$ and minimum $\left(\mathrm{MIN} ;-40.79^{\circ}\right)$ ).

The RMSE of the prediction model is $6.29^{\circ}$. Figure 6 shows the RMSE and the recorded versus the estimated MCP abduction/adduction angles, differentiating between grasp types.

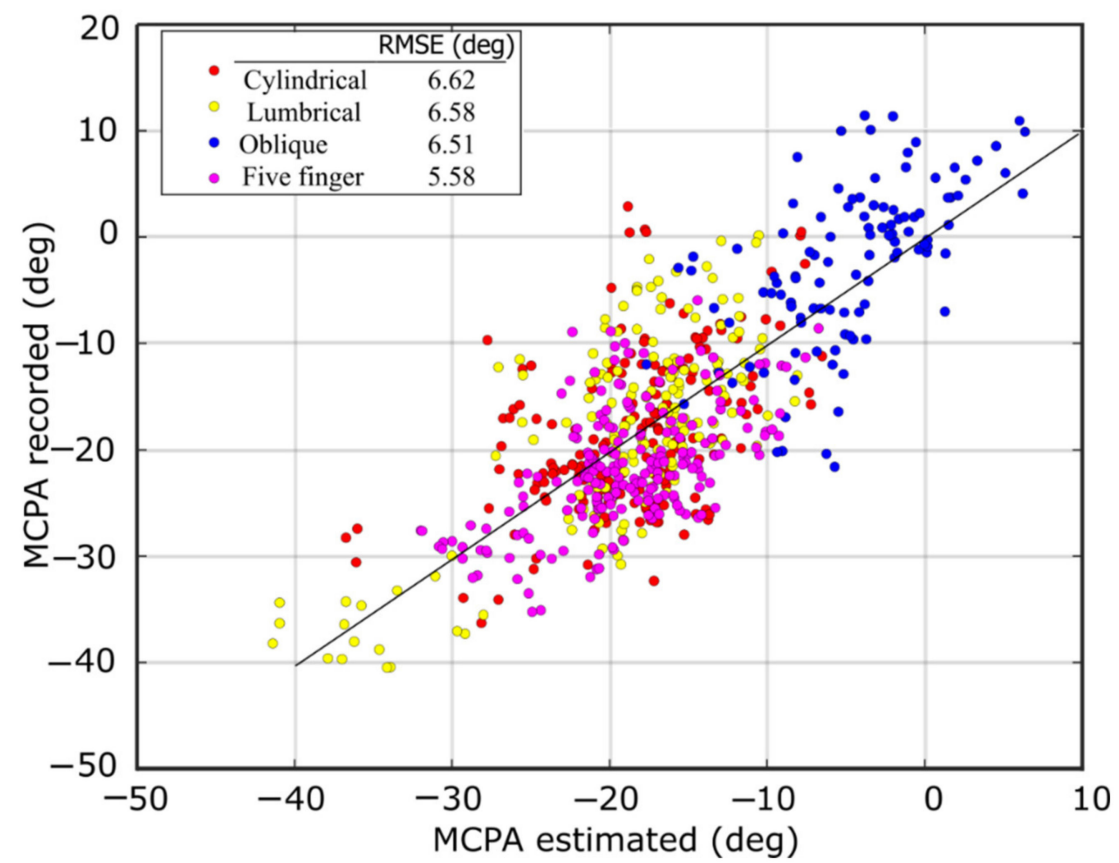

Figure 6. Root mean square error (RMSE) and recorded MCP abduction/adduction (MCPA) angles versus the estimated MCPA angles during all the postures recorded. 
Figure 7 shows the results of ANOVA conducted to check for significant differences in the errors for each grasp type, as well as some statistics (average $\mu$ and standard deviation $\sigma$ ) of the errors. The ANOVA presented a p-value of 0.39 , which indicates that differences in the estimated and recorded angles between grasps are not significant, with a power of 0.72 .

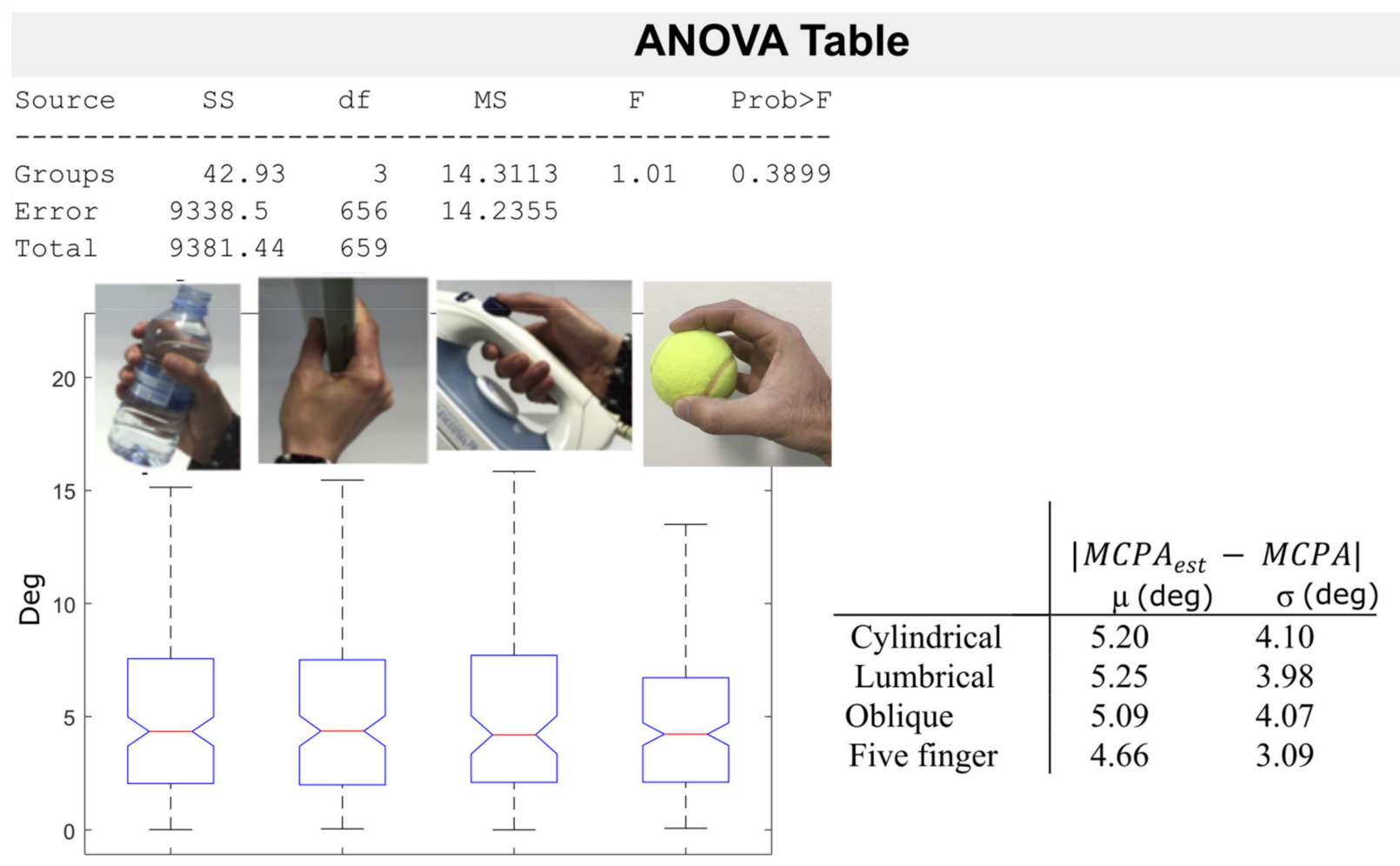

Cylindrical Lumbrical Oblique Five finger

Figure 7. ANOVA results and statistics (average $(\mu)$ and standard deviation $(\sigma)$ ) between estimated and recorded MCP abduction/adduction (MCPA) joint angles. Box plots are also provided for a visual comparison of the group location parameters. The horizontal mark in the center of the boxes is the median; the edges of the boxes are the 25th and 75 th percentiles; whiskers extend to 1.5 times the interquartile range.

\subsection{Experiment B. Evaluation of the Goodness of the Regression}

Figure 8 shows the recorded versus the estimated MCP abduction/adduction angles, differentiating between subjects (each subject is presented in a different color) for both datasets. The global RMSE (across subjects and frames/postures) presents values of 5.58 degrees (10.74\% with respect to the range of motion of MCP abduction/adduction) during reach-to-grasp movement, and 4.91 degrees $(9.45 \%$ with respect to the range of motion of MCP abduction/adduction) during grasping postures. 
(a)

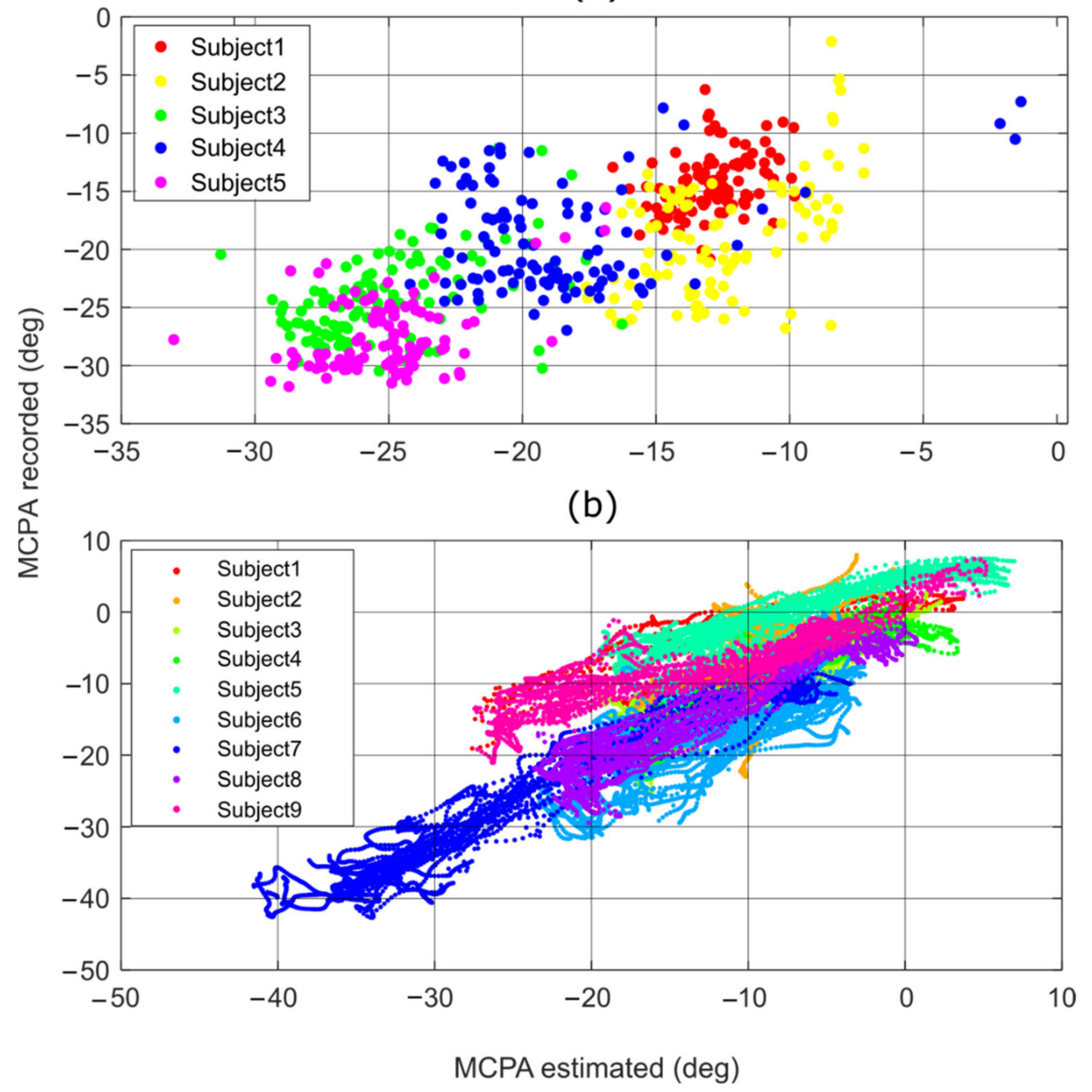

Figure 8. Recorded MCP abduction/adduction (MCPA) angles versus the estimated MCPA angles during all the recorded frames/postures from set $1(\mathbf{b})$ and set 2 (a). Subjects are presented in different colors.

\section{Discussion}

In this study, the correlation between thumb joints during grasps was explored, and a prediction model was obtained that allows the thumb MCP abduction/adduction angle to be estimated from the other joint motions of the thumb during grasp postures and free movements.

Several correlations between thumb joints were found, in which MCP abduction/ adduction presented the highest values: high correlation with CMC abduction/adduction (0.68), correlation with MCP flexion/extension (0.27), as well as negative correlation with CMC flexion/extension angles $(-0.30)$. The results are in accordance with the previous literature. CMC-MCP correlation was previously derived analytically, since abductor/adductor muscles for the CMC joints are also abductors/adductors for the MCP joint [4]. The CMCIP joint coordination is also present in previous studies [8] and reflects the fact that grasping, in general, requires flexing the joints to place the tip of the thumb in the grasping position. MCP flexion/extension appears poorly coordinated with the rest of the thumb joints. This result is novel with respect to the literature, in which it usually appears highly correlated with CMC flexion/extension movement during free actions such as circumduction and opposition movements [8]. These differences could be due to the fact that the activities used in our study are postures and not free movements, and therefore the coordination observed may be more linked to the object to be grasped (shape, weight, rigidity, etc.). 
The model used to estimate the abduction/adduction of the thumb MCP joint obtained low estimation error (6.29 degrees), with no significant differences between grasps. In our model, MCP abduction/adduction movement is expressed mainly from CMC joint movements, which is in accordance with the literature. Due to the action of the muscles that act on these joints, the thumb MCP joint is closely linked to the movements of the thumb CMC joint $[4,8]$. The interaction between CMC joint movements also has a physiological meaning [18]: depending on the thumb position (more/less flexed or more/less abducted), thumb ligaments are distended or not, thereby allowing or blocking the MCP abduction/adduction movement.

To the best of our knowledge, no previous studies have been conducted to obtain a prediction model for the MCP abduction/adduction joint movement as obtained herein. The model has been validated with other kinematic data with different grasp postures as well as during free movement (reach-to-grasp), with low estimation errors being obtained (ranging from 4.91 degrees to 5.58 degrees). The results could benefit many fields such as virtual reality or teleoperation, where many devices used to measure the kinematics of the hand (such as instrumented gloves) [19] do not typically include this joint movement. Since independent control of thumb joint motions affects the overall performance of the hand, synergistic approaches can provide viable solutions to simplify thumb models and for the design of prostheses and exoskeletons [7]. In this sense, biomechanical models, prostheses and exoskeletons may also benefit from it, by providing more accurate thumb joint positioning that it is important to incorporate for accurate thumb-tip force reproduction [7].

Author Contributions: J.L.S.-B. and M.V. conceived the study, designed the experiments and contributed to the writing phase. N.J.J.-B. performed the experiments and data analysis, as well as the writing phase. All authors have read and agreed to the published version of the manuscript.

Funding: This research was funded by the project PGC2018-095606-B-C21 (MCIU/AEI/FEDER, UE).

Institutional Review Board Statement: The study was conducted in accordance with the guidelines of the Declaration of Helsinki, and approved by the Ethics Committee of Universitat Jaume I (CD/31/2019, 6th June 2019).

Informed Consent Statement: Informed consent was obtained from all the subjects involved in the study.

Data Availability Statement: The data presented in this study are openly available in Zenodo at https:/ / doi.org/10.5281/zenodo.4646797 (accessed on 1 April 2021).

Conflicts of Interest: The authors declare no conflict of interest.

\section{References}

1. Cotugno, G.; Althoefer, K.; Nanayakkara, T. The Role of the Thumb: Study of Finger Motion in Grasping and Reachability Space in Human and Robotic Hands. IEEE Trans. Syst. Man Cybern. Syst. 2016, 47, 1061-1070. [CrossRef]

2. Edmunds, J.O. Current concepts of the anatomy of the thumb trapeziometacarpal joint. J. Hand Surg. Am. 2011, 36, 170-182. [CrossRef] [PubMed]

3. Kuroiwa, T.; Fujita, K.; Nimura, A.; Miyamoto, T.; Sasaki, T.; Okawa, A. A new method of measuring the thumb pronation and palmar abduction angles during opposition movement using a three-axis gyroscope. J. Orthop. Surg. Res. 2018, 13, 1-8. [CrossRef] [PubMed]

4. Brand, P.W.; Hollister, A.M. Clinical Mechanics of the Hand, 3rd ed.; Mosby Publishing: St. Louis, MO, USA, 1999.

5. Hollister, A.; Buford, W.L.; Myers, L.M.; Giurintano, D.J.; Novick, A. The axes of rotation of the thumb carpometacarpal joint. J. Orthop. Res. 1992, 10, 454-460. [CrossRef] [PubMed]

6. Grinyagin, I.V.; Biryukova, E.V.; Maier, M.A. Kinematic and dynamic synergies of human precision-grip movements. J. Neurophysiol. 2005, 94, 2284-2294. [CrossRef] [PubMed]

7. Nanayakkara, V.K.; Cotugno, G.; Vitzilaios, N.; Venetsanos, D.; Nanayakkara, T.; Sahinkaya, M.N. The Role of Morphology of the Thumb in Anthropomorphic Grasping: A Review. Front. Mech. Eng. 2017, 3, 5. [CrossRef]

8. Li, Z.-M.; Tang, J. Coordination of thumb joints during opposition. J. Biomech. 2007, 40, 502-510. [CrossRef] [PubMed]

9. Jarque-Bou, N.J.; Vergara, M.; Sancho-Bru, J.L.; Gracia-Ibáñez, V.; Roda-Sales, A. A calibrated database of kinematics and EMG of the forearm and hand during activities of daily living. Sci. Data 2019, 6, 270. [CrossRef] [PubMed] 
10. Della Santina, C.; Bianchi, M.; Averta, G.; Ciotti, S.; Arapi, V.; Fani, S.; Battaglia, E.; Catalano, M.G.; Santello, M.; Bicchi, A. Postural hand synergies during environmental constraint exploitation. Front. Neurorobot. 2017, 11, 41. [CrossRef] [PubMed]

11. Ciotti, S.; Battaglia, E.; Carbonaro, N.; Bicchi, A.; Tognetti, A.; Bianchi, M. A synergy-based optimally designed sensing glove for functional grasp recognition. Sensors 2016, 16, 811. [CrossRef] [PubMed]

12. Bullock, I.M.; Borras, J.; Dollar, A.M. Assessing assumptions in kinematic hand models: A review. In Proceedings of the IEEE RAS and EMBS International Conference on Biomedical Robotics and Biomechatronics, Rome, Italy, 24-27 June 2012; pp. 139-146.

13. Cobos, S.; Ferre, M.; Aracil, R. Simplified human hand models based on grasping analysis. In Proceedings of the IEEE/RSJ 2010 International Conference on Intelligent Robots and Systems, Taipei, Taiwan, 18-22 October 2010.

14. Vergara, M.; Sancho-Bru, J.L.; Gracia-Ibáñez, V.; Pérez-González, A. An introductory study of common grasps used by adults during performance of activities of daily living. J. Hand Ther. 2014, 27, 1-28. [CrossRef] [PubMed]

15. Sancho-Bru, J.L.; Jarque-Bou, N.J.; Vergara, M.; Pérez-González, A.; Perez-Gonzalez, A. Validity of a simple videogrammetric method to measure the movement of all hand segments for clinical purposes. Proc. Inst. Mech. Eng. H 2014, 228, 182-189. [CrossRef] [PubMed]

16. Hair, J.F.; Black, W.C., Jr.; Barry, J.B.; Anderson, E.R. Multivariate Data Analysis, 7th ed.; Prentice Hall: Hoboken, NJ, USA, 2009; ISBN 9780138132637.

17. Gracia-Ibáñez, V.; Vergara, M.; Sancho-Bru, J.L.; Mora, M.C.; Piqueras, C. Functional range of motion of the hand joints in activities of the International Classification of Functioning, Disability and Health. J. Hand Ther. 2017, 30, 337-347. [CrossRef] [PubMed]

18. Kapandji, I.A. The Physiology of the Joints, Volume 1: Upper Limb, 6th ed.; Churchill Livingstone/Elsevier: Amsterdam, The Netherlands, 2007.

19. Cui, L.; Cupcic, U.; Dai, J.S. Kinematic mapping and calibration of the thumb motions for teleoperating a humanoid robot hand. In Proceedings of the ASME Design Engineering Technical Conference, Washington, DC, USA, 28-31 August 2011. 